\title{
Identification of sequential auditory and visual stimuli'
}

ARTHUR S. KAMLET

UNIVERSITY OF MICHIGAN

\begin{abstract}
Two Ss identified pairs of $15 \mathrm{msec}$. one-bit auditory and visual stimuli when the interval between the stimuli varied from 0 to $500 \mathrm{msec}$. The auditory judgments were better when the auditory and visual signals were separated by $500 \mathrm{msec}$. than when the two signals were presented simultaneously, and were also better when the auditory stimulus followed the visual stimulus than when the visual stimulus was delayed.
\end{abstract}

\section{Problem}

Many authors have found that a decrement in performance results from information-processing tasks where non-redundant signals are presented simultaneously or nearly-simultaneously to two different sensory channels, when compared with signals presented to only one channel, or presented sequentially to the two channels. Kristofferson (1965), using pairedauditory and visual stimuli to test a channel-switching model of attention, found that for most of his Ss a scanning model, which predicts that "switching of attention is controlled by a periodic mechanism, and that switching can occur only once every M msec." was supported.

Lindsay et al (1965), using an absolute judgment task with auditory and visual stimuli, presented either singly to one modality, or simultaneously to both modalities, and with stimulus durations of either $50 \mathrm{msec}$. or $2 \mathrm{sec}$., found that judgments in each modality were superior for the single presentation case. They did not find any

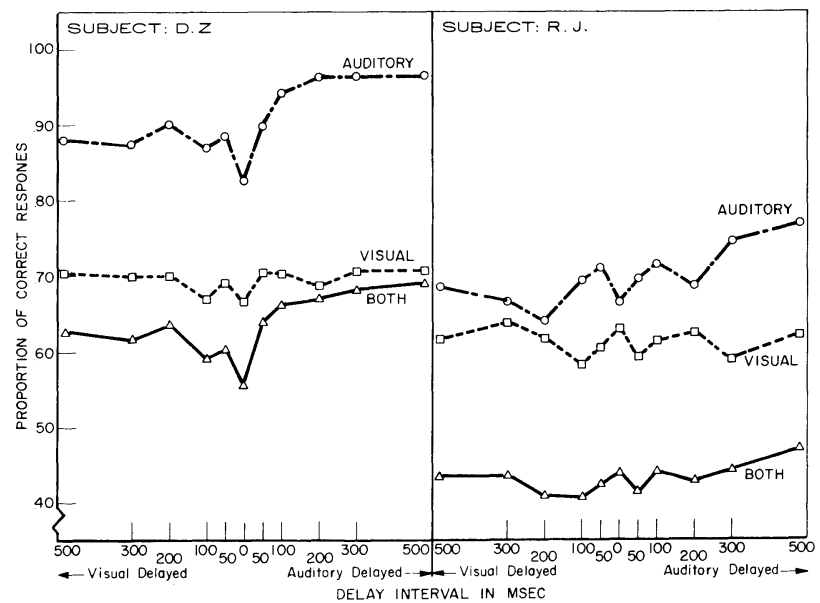

Fig. 1. Proportion of correct responses as a function of delay interval. interaction between single vs. simultaneous presentation and stimulus duration, and point out that if there was switching, "it occurred with approximately equal effects for both the long and short stimuli." They suggest that if a sufficiently long perceptual trace of the $50 \mathrm{msec}$. stimulus was available, switching still could have occurred.

If one-bit absolute judgments are made about brief auditory and visual stimuli, while the interval between the stimuli is varied from zero-simultaneous presentation-to some value larger than $M$, the function relating identification of each stimulus to the duration of this interval can be predicted from a scanning model of channel switching with a period of $\mathrm{M}$ msec. Identification should be minimal for simultaneous presentation, should reach an asymptotic maximal value at $M$ and, assuming a uniform distribution of switching times, should linearly increase as the interval varies from zero to $M$. Method

Each trial consisted of either a bright (103 ft-L) or a $\operatorname{dim}(88 \mathrm{ft}-\mathrm{L}) 15 \mathrm{msec}$. light-flash and either a high (1900 pulse/sec.) or a low (1800 pulse/sec.) pitched $15 \mathrm{msec}$. tone-burst, and S's task was to identify, with whatever latency and in any order he chose, both the visual and auditory signals. Immediately following the second identification feedback was provided. To prevent S from "ignoring" either modality, on every trial he was given a payoff of $1 / 2$ cent, $1 / 8$ cent and 0 cents respectively, for both responses correct, only one response correct, and neither response correct.

Sessions consisted of 12 blocks of 100 trials, each lasting approximately $6 \mathrm{~min}$. Each $\mathrm{S}$ ran for one practice and 11 experimental sessions in accordance with a randomized Latin square design. The 12 blocks consisted of 5 delay intervals $-50,100,200,300$, and $500 \mathrm{msec}$.by 2 orders of presentation-visual or auditory modality delayed. The first block each day was a practice block, and there was one block of simultaneously presented stimuli. In any block of trials each stimulus-pair occurred equally often and in a random order within the block.

The Ss were two male graduate students with experience in making psychophysical judgments.

\section{Results}

For each delay interval the proportions of correct identifications of the auditory stimulus, the visual stimulus, and both the auditory and the visual stimuli were calculated for each $\mathrm{S}$, and the results, averaged over 11 sessions, are shown in Fig. 1. For each S, 
Chi-squared contingency tests $(d f=10)$ showed that there is a significant relationship between delay interval and identification of the auditory stimulus $(p<.001)$, but the relationship between delay and visual identification did not approach significance. The auditory results were not symmetrical about the zero delay point, but indicated that identification was better when the auditory stimulus was delayed by $300-500 \mathrm{msec}$. than when it preceded the visual stimulus by this amount $(p<.001)$. For both Ss auditory identification is significantly poorer when the two stimuli are presented simultaneously than when the auditory stimulus is delayed by $300-500 \mathrm{msec} .(p<.001)$ and for one $S$ (D. Z.) this is also true when the visual stimulus is delayed.

If the assumption of independent processing of information from the two sensory channels is correct, a necessary though not sufficient condition which mustbe met for each delay interval, is that the probability of a correct identification on both channels be equal to the product of the probabilities of correctidentifications on the separate channels. A Chi-squared test $(d f=10)$ between the obtained values and those predicted by the independent probabilities assumption at each delay interval yielded values of 0.15 and 0.012 for R. J. and D. Z., respectively, indicating rather small differences.

\section{Conelusions}

The data for D. Z a at first seem to be described by a channel-switching model with a switching period of approximately $100 \mathrm{msec}$. This can be misleading, however, for as Kristofferson has pointed out, the S must have the sensory channel of the first occurring signal "aligned" with attention, otherwise switching times will be overestimated. In the present study, before each block of trials, $\mathrm{S}$ was told which modality would occur first, but this does not assure that attention was aligr ith the proper channel. Furthermore, even when the stimuli were presented simultaneously, performance was far above chance level. This result, also found by Lindsay et al, might indicate either the existence of a perceptual trace of the signals, or that there may be more than one channel attended to at one time.

The asymmetry noted in the data might be due to an auditory trace that fades more quickly than the visual trace, or which is masked more by the visual signal than the visual trace is masked by the auditory signal. A longer lasting visual trace would explain why the delay interval had no significant effect on visual identification.

While the data do not provide unambiguous support for a channel-switching theory, it is clear that a delay of only $300 \mathrm{msec}$. between very simple visual and auditory signals will yield better auditory performance than when the two signals are presented simultaneously. This is in agreement with, and an extension of a statement by Lindsay et al "that two attentive acts cannot be performed simultaneously without some loss in efficiency."

\section{References}

Kristofferson, A. B. Attention in time discrimination and reaction time. NASA CR-194, 1965.

Lindsay, P. H., Cuddy, Lola L., \& Tulving, E. Absolute judgments of simultaneously presented visual and auditory stimuli. Psychon. Sci., 1965, 2, 211-212.

\section{Note}

1. This research was supported in part by the Advanced Research Projects Agency, Department of Defense and was monitored by the Air Force Office of Scientific Research under Contract No. AF 49 (638)-1235 with the Human Performance Center and was conducted while the author held a Link Foundation fellowship. 